Chirurg 2013 $\cdot 84: 329$

DOI 10.1007/s00104-013-2493-0

Online publiziert: 13. März 2013

๑) Springer-Verlag Berlin Heidelberg 2013
O. Strobel · M.W. Büchler

Klinik für Allgemein-, Viszeral- und Transplantationschirurgie, Universität Heidelberg

\section{Leberzirrhose mit akuter Ösophagusvarizenblutung}

\section{TIPS vs. chirurgischer portokavaler Shunt}

\section{Originalpublikation}

Orloff MJ, Vaida F, Haynes KS et al (2012) Randomized controlled trial of emergency transjugular intrahepatic portosystemic shunt versus emergency portacaval shunt treatment of acute bleeding esophageal varices in cirrhosis. J Gastrointest Surg 16:2094-2111

\section{Hintergrund und Fragestellung}

Die Ösophagusvarizenblutung (ÖVB) bei Leberzirrhose ist mit einer hohen Mortalität assoziiert. Eine optimale Therapie ist daher von zentraler Bedeutung. Bei fehlender Blutungskontrolle durch eine medikamentöse und endoskopische Therapie hat die transjuguläre intrahepatische portosystemische Shunt-Anlage (TIPS) die früher übliche chirurgische portokavale Shunt-Anlage (PCS) weitgehend verdrängt. Vergleichende Studien zu den Ergebnissen beider Verfahren fehlen allerdings weitgehend. Orloff et al. verglichen in einer randomisiert kontrollierten Studie die Ergebnisse von TIPS und PCS in der Notfalltherapie von Patienten mit Leberzirrhose und akuter ÖVB.

\section{Methoden}

Insgesamt 154 unselektionierte Patienten mit Leberzirrhose und akuter ÖVB wurden in die Gruppen TIPS ( $\mathrm{n}=78$ ) und PCS $(\mathrm{n}=76)$ randomisiert. TIPS oder PCS wurden innerhalb von $24 \mathrm{~h}$ nach Aufnahme durchgeführt. Für die TIPS-Anlage wurden nicht-PTFE(Polytetrafluorethylen)beschichtete Stents verwendet. Die PCSAnlage erfolgte als portokavale Seit-zuSeit-Anastomose bei 72 Patienten und als End-zu-Seit-Anastomose bei 5 Patienten.
Als wichtigste Zielparameter wurden Blutungskontrolle, Offenheitsrate, Enzephalopathie und Überleben analysiert.

\section{Ergebnisse}

Die Gruppen waren bezüglich epidemiologischer Parameter, der Ätiologie und Schwere der Leberzirrhose (20\% Child A, 50\% Child B, 30\% Child C) und der Schwere der ÖVB vergleichbar. TIPS führte in $80 \%$ zur Blutungskontrolle für die ersten 30 Tage, aber nur in $22 \%$ zur Langzeitkontrolle. Die chirurgische PCSAnlage führte zur primären Blutungskontrolle in $100 \%$ und zur permanenten Kontrolle in $97 \%(\mathrm{p}<0,001)$. TIPS-Patienten benötigten fast doppelt so viele Bluttransfusionen wie PCS-Patienten. Nach TIPS kam es bei $84 \%$ der Patienten zu einer Stenose/Okklusion, während die Offenheitsrate nach PCS 97\% betrug. Die Überlebensraten waren über alle Zeitintervalle und Child-Stadien nach PCS höher als nach TIPS. Das mediane Überleben betrug mehr als 10 Jahre nach PCS und nur 1,99 Jahre nach TIPS $(\mathrm{p}<0,001)$. Nach PCS entwickelten $21 \%$ der Patienten, nach TIPS 61\% der Patienten eine Enzephalopathie $(\mathrm{p}<0,001)$.

\section{Diskussion}

Die Autoren folgern, dass die chirurgische PCS-Anlage der TIPS-Anlage mit einem nicht-PTFE-beschichteten Stent überlegen ist. Anhand der schlechten Ergebnisse der TIPS und der sehr guten Ergebnisse der PCS schlagen die Autoren die chirurgische PCS-Anlage als Erstlinientherapie in der Behandlung der akuten Varizenblutung bei Leberzirrhose vor.
Bei der Bewertung dieser Studie müssen folgende Punkte beachtet werden: - In der Studie wurden nicht die mittlerweile üblichen (e-PTFE-)beschichteten Stents verwendet, die möglicherweise zu besseren Offenheitsraten und daher auch zu besseren Langzeitergebnissen nach TIPS führen.

- Die hervorragenden Langzeitergebnisse nach PCS stammen aus einem Zentrum mit langjähriger hoher Expertise in der chirurgischen PCS-Anlage und sind nur aufgrund der hohen primären Erfolgsrate und Offenheitsrate der PCS sowie durch eine lebenslange, enge Anbindung der Patienten an das Zentrum möglich. Diese Ergebnisse sind nicht unbedingt auf andere Techniken der portosystemischen Shunt-Anlage und auf andere Institutionen übertragbar.

Sicher ist allerdings, dass mit der chirurgischen PCS-Anlage überzeugende Langzeitergebnisse erreicht werden können, die für die TIPS so noch nicht gezeigt wurden. Basierend auf diesen Daten sollte die Indikation zur chirurgischen PCS-Anlage wieder häufiger gestellt werden.

\section{Korrespondenzadresse}

\section{PD Dr. O. Strobel}

Klinik für Allgemein-, Viszeral- und Transplantationschirurgie, Universität Heidelberg,

Im Neuenheimer Feld 110, 69120 Heidelberg Oliver.Strobel@med.uni-heidelberg.de

Interessenkonflikt. Der korrespondierende Autor gibt für sich und seinen Koautor an, dass kein Interessenkonflikt besteht. 